Manuelle Medizin 2022 $\cdot 60: 1-2$

https://doi.org/10.1007/s00337-021-00854-y

Angenommen: 20. Dezember 2021

(C) The Author(s), under exclusive licence to Springer Medizin Verlag GmbH, ein Teil von Springer Nature 2022

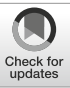

\title{
Rückblick auf das Coronajahr 2021
}

\author{
L. Beyer ${ }^{1} \cdot$ U. Smolenski \\ 'Ärztehaus Mitte, Jena, Deutschland \\ ${ }^{2}$ Friedrich-Schiller-Universität, Institut für Physiotherapie, Jena, Deutschland
}

\section{Virtueller Austausch und kontinuierliche Fortbildung \\ Liebe Leserinnen! \\ Liebe Leser!}

Unter den vielen ungünstigen Aspekten, die das Auftreten des Coronavirus für uns mit sich gebracht hat, gibt es vielleicht auch einen positiven Aspekt, wenn man optimistisch auf die Dinge schaut.

Die internationale Gemeinschaft der Manuellen Medizin (FIMM) führte bereits zum zweiten Male eine wissenschaftliche Konferenz als Zoom-Meeting durch. Stephan Vinzelberg aus Berlin war als Vertreter der Deutschen Gesellschaft (DGMM) in der FIMM maßgeblich an der Organisation beteiligt. Bei solchen Konferenzen im virtuellen Raum fallen zwar die persönlichen Begegnungen und Gespräche in den Pausen und Abenden der Veranstaltung weg, andererseits aber können sich Teilnehmer aus aller Welt treffen, die vielleicht sonst nicht die Möglichkeit gehabt hätten, die Vorträge anzuhören bzw. anzusehen. Das nächste virtuelle wissenschaftliche Kolloquium soll am 19. März 2022 stattfinden. Alle Leser unserer Zeitschrift können sich bei Interesse unter www.fimm-online.com informieren.

\section{European Core Curriculum und Prinzipien der Manuellen Medizin verabschiedet}

Am 18. September 2021 verabschiedete die ESSOMM-Vollversammlung das ESSOMM European Core Curriculum und Prinzipien der Manuellen Medizin.

In zehn 3-tägigen Jahrestreffen, die zwischen 2010 und 2021 in Rom stattfanden, erarbeitete die European Scientific Society of Manual Medicine (ESSOMM) einen Konsens über ein Curriculum und die Prinzipien der Manuellen Medizin, basierend auf translationaler Forschung, klinischen Studien und den klinischen Erfahrungen der verschiedenen nationalen Fachvertreter. Viele Autoren aus allen Mitgliedsländern haben wesentlich zu den wichtigen Themen beigetragen, die diskutiert und vereinbart werden sollten. Es war ein anspruchsvoller und langwieriger Prozess, alle Fragen dieses Vorhabens zufriedenstellend zu beantworten, aber letztendlich wurde dies erreicht. Das vorliegende Papier wird in unserer Zeitschrift veröffentlicht werden.

Heute bilden 14 nationale wissenschaftliche Gesellschaften mit mehr als 12.000 Ärzten die ESSOMM (www. essomm.eu).

\section{Weitere Veranstaltungen}

Die Internationale Akademie für Manuelle Muskuloskeletale Medizin (IAMMM www.iammm.net) hat es nach einem Jahr Pause wieder gewagt, ihre Tagung in Präsenz durchzuführen. Tagungsort war das anatomische Institut der Universität Padua, die in diesem Jahr ihren 800. Gründungstag feiern kann. Die Tagung wurde vor Ort in Kooperation mit der bekannten Faszienforscherin Carla Stecco organisiert. Coronabedingt hielt sich die Teilnahme mit ca. 40-50 Teilnehmern in Grenzen. Unsere Leser können sich aber in dieser Ausgabe unserer Zeitschrift über die Inhalte der Beiträge anhand der Abstracts informieren. Vertreter der DGMM präsentierten 4 Beiträge. 
Die Deutsche Gesellschaft für Physikalische und Rehabilitative Medizin führte ihren Jahreskongress ebenfalls virtuell durch. Mehrere Beiträge waren manualmedizinisch orientierten Inhalten gewidmet. Besonderen Anklang fanden der Vortrag und Demonstrationen zu Selbstübungen am Arbeitsplatz von Norman Best.

Trotz alledem kann nicht verschwiegen werden, dass die verschiedenen Coronamaßnahmen den Kursbetrieb in der Fortbildung Manuelle Medizin beträchtlich behindert haben.

\section{Beiträge dieses Heftes}

Über „Präsenzkurse unter Lockdownbedingungen-eine Herausforderung" berichten A. Sammer et al. Aus einer retrospektiven Befragung der Teilnehmer, ob es innerhalb der ersten 2 Wochen nach Kursende zu Krankheitssymptomen gekommen ist, erfahren wir, dass kein Kursant sich während der Kurse mit SARS-CoV 2 infiziert hat. Die Entwicklung und Umsetzung eines entsprechenden Sicherheitskonzepts, das den gesetzlichen Maßstäben gerecht werden muss, ermöglicht auch in Zeiten harter Hygieneauflagen einen Kursbetrieb. Das Interesse an Fortbildung in Manueller Medizin ist ungebrochen.

Weitere Inhalte dieses Heftes sind die Ergebnisse einer Interventionsstudie im Messwiederholungsdesign mit Kontrollgruppe von Ch. Anders et al.: „Effekt von Gangjustierhilfen auf die Stabilisierung und Symmetrisierung des Gehens".

Besonders aufmerksam machen möchten wir Sie auf den Beitrag "Mentale Gesundheit und physische Aktivität" von W. Laube, wenn Sie sich für die muskulo-, neuro- und immunphysiologischen Hintergründe von körperlicher Aktivität und Training, wie es auch in der Manuellen Medizin Patienten empfohlen wird, interessieren. Hier gibt es auch eine funktionelle Verbindung zu den Mechanismen der Schmerzwahrnehmung: „Myokine der aktiven Muskulatur prägen die Entwicklung und Erhaltung des Gehirns wesentlich mit. Kognitive Leistungen werden gefördert. Inaktivität hat gegenteilige Wirkungen. Das psychophysische Leistungsverhalten leidet und es kann eine Disposition für zentrale Sensibilisierungen entstehen. Die Muskelaktivität ist das Fundament für einen guten Funktionsstatus des Gehirns selbst sowie der peripheren Gewebe und Organe."

\section{CME}

Ärztliche Fortbildung wurde bereits Ende des 19. Jahrhunderts initiiert und landesweit von nicht Geringeren wie Ernst von Bergmann, Robert Koch und Rudolf Virchow organisiert. Also eine Institution, der sich die deutsche Ärzteschaft verpflichtet fühlt, gerade in einer Zeit des Wandels der Lehre. Nicht nur durch Corona, sondern durch moderne Medien und Kommunikationsmittel ergeben sich verschiedene Möglichkeiten der Wissensvermittlung.

"Continuing medical education" (CME) enthält evidenzbasiertes Wissen zur Vermittlung und Festigung von Kenntnissen, Aktivitäten und professionellem Verhalten, die wir Ärzte für die tägliche Arbeit brauchen. Sie vermittelt gebietsbezogene Inhalte und interdisziplinäres Wissen für die klinische Arbeit - insbesondere in einem Querschnittsbereich wie der Manuellen Medizin unerlässlich. Flexibles Lernen ist inhaltlich und methodologisch beschrieben als mediengestütztes Eigenstudium (Fachliteratur, audiovisuelle Lehrund Lernmittel, strukturierte interaktive Fortbildung); diesem sollen in Manuelle Medizin CME-Beiträge aus den eigenen Reihen und mit Kenntnissen der anderen Fachgebiete zukünftig mehr gerecht werden. Wir hoffen auf reges Interesse und sind für Wünsche offen.

\section{Korrespondenzadresse}

\section{Beyer}

Ärztehaus Mitte

Westbahnhofstr. 2, 07745 Jena, Deutschland lobeyer@t-online.de

Interessenkonflikt. L. Beyer und U. Smolenski geben an, dass kein Interessenkonflikt besteht.

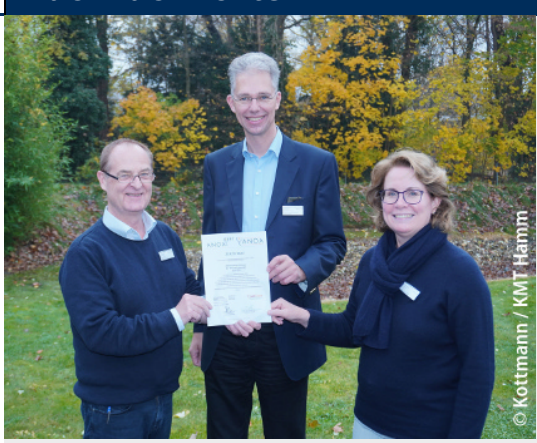

Qualitätssiegel für Klinik in St. Wendel

Das Marienhausklinikum St. WendelOttweiler ist am 19. November 2021 auf der ANOA-Strategietagung in Hamm mit dem Qualitätssiegel ANOA-Cert 2.0 ausgezeichnet worden.

Mit dem 2016 entwickelten ANOA-Zertifikat können Kliniken ihre Struktur-, Prozessund Ergebnisqualität nachweisen und sichern. Nach der erfolgreichen Überarbeitung des ANOA-Konzeptes im Jahr 2020 wurde auch dieses Qualitätssicherungsund Zertifizierungssystem aktualisiert. In seiner neuen Fassung wurde es mit dem nun abgeschlossenen Zertifizierungsprozess in der Klinik in St. Wendel-Ottweiler erstmals zur Anwendung gebracht.

Mit der erneuten Vergabe des Qualitätssiegels an die Klinik des Chefarztes Dr. Jan Holger Holtschmit (zugleich Präsident der ANOA) können bundesweit nun weiterhin sieben Kliniken mit dem Qualitätssiegel des ANOA-Verbundes werben.

Die ANOA (Arbeitsgemeinschaft nicht operativer orthopädischer manualmedizinischer Akutkliniken) ist eine medizinisch-wissenschaftliche Vereinigung von mittlerweile 32 Akutkrankenhäusern, die im nicht operativen orthopädisch-unfallchirurgischen, manualmedizinischen und schmerztherapeutischen Bereich tätig sind.

Quelle: ANOA, anoa-kliniken.de 